\title{
Active Videogames to Promote Traditional Active Play: Increasing the Reinforcing Value of Active Play Among Low-Active Children
}

\author{
Kelsey Ufholz, PhD, ${ }^{1}$ Kyle D. Flack, PhD, RD, ${ }^{1,2}$ LuAnn Johnson, MS, ${ }^{1}$ and James N. Roemmich, PhD ${ }^{1}$
}

\begin{abstract}
Background: Exercise reinforcement predicts physical activity of children. Repeated exposures of physical activity may increase physical activity reinforcement (incentive sensitization). Active videogame (AVG) play produces light-to-moderate-intensity physical activity. Ideally, AVG play would transition to nonscreen-based active play through incentive sensitization of traditional active play (TAP), providing AVG does not increase sedentary videogame (SVG) reinforcement. Greater autonomy increases motivation toward traditional physical activity, but whether autonomy enhances incentive sensitization has not been studied.

Objectives: To determine whether autonomy over AVG play promotes incentive sensitization of TAP or SVG. Methods: Inactive children (ages 8-12; 5th-97th body mass index percentile) were provided with AVG and SVG for 6 weeks and assigned to either a high autonomy $(n=25)$ or low autonomy $(n=24)$ group, differentiated by AVG choice and more freedom over amount of play. Both groups played AVG 3 days per week. SVG were played ad libitum. Participants completed an operant responding task to measure the relative reinforcing value (RRV) of AVG versus $S V G\left(R_{\text {RV }}\right.$ AVG vs. SVG) and AVG versus TAP ( $\left.R R V_{\text {AVG vs. TAP }}\right)$ at baseline, 6 weeks, and 10 weeks. Results: $\mathrm{RRV}_{\mathrm{AVG}}$ vs. $\mathrm{SVG}$ increased over time $(P=0.056)$ but did not differ by autonomy or autonomy $\times$ time $(P=0.184)$. RRV $\mathrm{AVG}$ vs. TAP decreased over time $(P=0.033)$ but did not differ by autonomy or autonomy $\times$ time $(P=0.73)$.

Conclusion: AVG play does not increase motivation toward SVG and increases motivation to play AVG relative to TAP. Providing higher autonomy did not promote incentive sensitization of play.
\end{abstract}

Keywords: Children, Physical activity, Relative reinforcing value, Videogames

Introduction

$\mathbf{C}$ URRENT HEALTH GUIDELINES recommend that children engage in exercise, sport, or active play for a minimum of 60 minutes per day. ${ }^{1}$ Despite this, sufficient physical activity is rare; as only $26.1 \%$ of children in 2017 engaged in the recommended amount. ${ }^{2}$ Alarmingly, this percentage further decreases during adolescence and into adulthood, ${ }^{3}$ increasing the risk of many later-onset illnesses. ${ }^{4}$ Encouraging greater physical activity in children is of great importance to both their current and future health.

Behavioral Choice Theory details that individuals have many competing options for how to spend their leisure time. ${ }^{5}$ One influential factor is the reinforcing value (RRV) of the alternatives. ${ }^{5}$ RRV may be operationalized as the amount of operant responding, that is, "work," a participant is willing to engage in to gain access to the desired "reward." This is often measured through an operant conditioning task measuring the relative amount of time an individual earns toward either physical activity and a competing sedentary alternative. The ratio of physical activity time earned to total time earned in both options is termed relative reinforcing value (RRV). This paradigm has been successfully applied to understand greater usual physical activity participation in both children ${ }^{6,7}$ and adults. ${ }^{8,9}$ If physical activity is to be maintained long-term, children must find it more reinforcing relative to a non-exercise (sedentary) alternative. This may be accomplished by either increasing the RRV of physical activity itself, termed incentive sensitization, or by decreasing the RRV of the sedentary behavior so that the RRV of physical activity increases relative to sedentary behavior (RRV).

\footnotetext{
${ }^{1}$ United States Department of Agriculture, Agricultural Research Service, Grand Forks, North Dakota.

${ }^{2}$ Department of Dietetics and Human Nutrition, University of Kentucky, Lexington, Kentucky.

(C) Kelsey Ufholz, et al., 2019; Published by Mary Ann Liebert, Inc. This Open Access article is distributed under the terms of the Creative Commons License (http://creativecommons.org/licenses/by/4.0), which permits unrestricted use, distribution, and reproduction in any medium, provided the original work is properly credited.
} 
A possible method of increasing physical activity of children is through playing active videogames (AVGs), a game system where the player's movements through the game space are displayed on a television screen. AVG may be ideal for promoting incentive sensitization of physical activity as children who engage in small amounts of physical activity may initially find AVG more reinforcing than traditional active play (TAP) by reducing concerns with coordination, sport-specific skills, social anxiety, and physical disabilities/ illnesses that may make participation in TAP difficult or aversive. ${ }^{10,11}$ AVG mimic TAP, such as sports, dancing, and obstacle courses, and in doing so, promote light-to-moderate physical activity in children. ${ }^{12}$ Studies of whether AVG play result in increased energy expenditure often show conflicting results, with some studies showing no increased energy expenditure over nonactive sedentary videogames (SVGs) ${ }^{13}$ and others showing increased physical activity. ${ }^{12,14}$ AVG energy expenditure appears to be particularly high in games facilitating lower body movements, such as running and kicking, ${ }^{12,15}$ but typically lower than occurs in TAP. ${ }^{16}$ Increased exposure to active play through AVG may promote incentive sensitization of AVG, TAP, or both; but this is not yet known. If AVG play produces greater increases in TAP reinforcement than AVG reinforcement, then children would be more likely to choose to try similar games in a more traditional context. Therefore, AVG play may act as a gateway to more TAP among children. ${ }^{17}$

While children find AVG enjoyable, often once the novelty wears off, their interest wanes and AVG play decreases. ${ }^{18}$ This is consistent with initial increase and subsequent decline of other exercise behavior in both adults and children. ${ }^{19}$ However, certain factors help maintain exercise motivation and behavior. Among them is exercise autonomy. ${ }^{20}$ Increasing perceived autonomy increases internal motivation for exercise $^{21}$ and predicts increases in short- ${ }^{22,23}$ and long-term exercise behavior. ${ }^{2425}$ Increasing children's autonomy by giving them increased choices of physical activities to partake in AVG play may make AVG play more reinforcing and enhance incentive sensitization of AVG and/or TAP, which may lead to greater physical activity. ${ }^{22,26}$

One of the most common alternatives to physical activity is screen-based sedentary activities, such as television, videogames, computer games, etc. These activities are very popular, with $65 \%$ of 4 - to 11-year-old children using these devices for more than 2 hours per day. ${ }^{27}$ Adolescents who play AVG are more likely to play SVGs, and engage in other sedentary screen-based behaviors such as watching television. $^{28}$ Therefore, it is equally possible that rather than increasing AVG or TAP reinforcement, children exposed to AVG may increase SVG reinforcement. This outcome would have the unintended consequence of increasing sedentary behavior, possibly enough to offset any activity gains by increasing AVG or TAP reinforcement.

This study examined AVG play in inactive children who were new to such games. We hypothesized that children who were given increased autonomy over their AVG play would show increased incentive sensitization toward AVG play, operationalized as a greater relative RRV. It was further hypothesized that exposure to AVG play would lead to increased reinforcement of both SVG play and TAP, relative to AVG play.

\section{Materials and Methods}

\section{Study designs}

This randomized controlled trial consisted of a two-group factorial design with autonomy condition treated as a betweensubject variable (greater autonomy, low autonomy), with low autonomy considered as the control group. Study assessments were conducted by trained research staff and took place at the Grand Forks Human Nutrition Research Center or their community-based laboratory in a local fitness center between November 2016 and April 2018. AVG play, the study treatment, took place in participants' homes. RRV $\mathrm{AVG}_{\mathrm{Av} \text {. SVG }}$ and $\mathrm{RRV}_{\text {AVG vs. TAP }}$ sessions took place on separate days, usually in the late afternoon or evening. This study received approval from University of North Dakota's Institutional Review Board and was registered at ClinicalTrials.gov (NCT02940431).

\section{Participants}

Participants were healthy inactive children (8-12 years of age). Participants were $51 \%$ female $(n=26)$. The majority identified as white $(76.9 \%, n=40)$, with minorities identifying as black $(1.9 \%, n=1)$, Asian $(7.7 \%, n=4)$, Hawaiian/ Pacific Islander $(1.9 \%, n=1)$, multiracial $(9.6 \%, n=5)$, and Hispanic $(1.9 \%, n=1)$. Of the 52 participants who entered the study, 1 withdrew before randomization, 2 withdrew after randomization, and 49 completed the study. Both nonoverweight and overweight children (5th-97th body mass index $[\mathrm{BMI}]$ percentile) were included. Entry criteria included not currently trying to lose weight, no medical conditions which might impede physical activity, not playing an organized sport or engaging in leisure time moderate-tovigorous physical activity more than three times per week for 1 hour at a time, not currently playing AVGs, and willingness to adhere to study treatments and measurement schedules. Families which indicated already owning an AVG system were permitted entrance in the study if they confirmed that their child used the AVG system for less than half an hour per week.

\section{Procedure}

Screening and consent visit. Initial screening took place online. The first in-person session included a review of answers to the online screening questions, study procedures, informed consent from the parent, and informed assent from the child. Parents were asked to provide demographic and health history information regarding their child. Children were measured for height and weight.

RRV assessments. Children were assessed for their

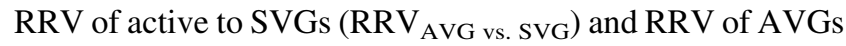
to traditional active games $\left(\mathrm{RRV}_{\mathrm{AVG}}\right.$ vs. TAP). Participants completed both RRV assessment tasks at baseline, 6 weeks (postintervention), and 10 weeks (postfollow-up). During this task, children were instructed to complete a computerbased task, in which they could earn minutes to be spent in an AVG, SVG, or TAP of their choice (see Measurements section). 


\section{Intervention}

Following baseline RRV assessments, families were provided with an $\mathrm{Xbox}^{\circledR} 360^{\mathrm{TM}}$ game system with the necessary accessories, including a Kinect ${ }^{\mathrm{TM}}$ motion detection system, which displays the player's outline on the television screen. Participants also received both AVG and SVG discs, with each disc containing a range of games and gaming options. AVG and SVG were chosen based upon popularity with the target demographic and age-appropriate ratings for 8- to 12year-old children. AVG required active play-like body movements, better to simulate traditional play, such as running, kicking, and dancing.

Participants in both groups were instructed to play AVG three times per week. Participants in the high autonomy groups were given two AVG discs of their choice and instructed to play these games for at least 20 minutes per session. Participants in the low autonomy group were assigned one AVG disc from among their top 3 most-liked games and instructed to play the game for 20-40 minutes per session. Both groups could play SVG as they chose but could not play any videogames except those provided by the research team. Parents were instructed to not place limits on their children playing SVG. Every 2 weeks, participants received a new set of videogame discs. Participants were instructed to record their videogame playing time on a log sheet.

Following 6 weeks of treatment, children completed the postintervention RRV and entered a 4-week washout period. During washout, participants in both groups were supplied with two AVG and two SVG of their choosing. Children could play both game types ad libitum, and any other videogames that they might already have at home. After 4 weeks of washout, children completed their final RRV assessments, identical to those at baseline and 6 weeks. Following the assessment, participants were debriefed, asked for intervention feedback, and thanked for their time. The child received $\$ 435$ and the parent $\$ 110$ as compensation.

\section{Measurements}

Height and weight. Body weight was measured through a Tanita scale. Participants wore light clothing and no shoes during measurement. Height was measured using a stadiometer. BMI percentile was calculated based upon growth curves for each participant's age and gender. ${ }^{29}$

Liking. Children were instructed to rate their liking of five AVG options (Kinect Dance 2015 ${ }^{\mathrm{TM}}$, Kinect Dance $2016^{\mathrm{TM}}$, Kinect Sports Season $1^{\mathrm{TM}}$, Kinect Sports Season $2^{\mathrm{TM}}$, and Kinect Adventures ${ }^{\mathrm{TM}}$ ) and five SVG options (Minecraft $^{\mathrm{TM}}$, Lego Avengers ${ }^{\mathrm{TM}}$, Lego Star Wars ${ }^{\mathrm{TM}}$, NHL Legacy ${ }^{\mathrm{TM}}$ edition, and NBA2K16 $\left.{ }^{\mathrm{TM}}\right)$ on a 10 -point scale $(1=$ do not like it at all, $10=$ like it very much). When earning time for AVG or TAP, children were asked to rate the liking of the same five AVG and three TAP (soccer, mini basketball, racquetball).

Twenty-four-hour recall. As a check on compliance and method of quantifying minutes of play, children were instructed to fill out a recall measure describing the previous day's activities. Children were instructed to fill out the recall for two randomly selected weekdays and two weekend days.
Recalls were completed at baseline, at weeks 2 and 4 of the intervention, after the intervention (6 weeks), and after washout (10 weeks). The children were asked to report hours and minutes spent in activities, such as AVG, SVG, and TAP, in addition to school, chores, traveling by car/bus, etc. The recall was based upon a recall diary used to measure AVG play in adolescents. ${ }^{30}$

Relative RRV and Pmax of AVG versus SVG. The most liked type of AVG and SVG activities were provided as alternatives for the RRV testing session. Participants played a slot machine-style game, in which three shapes of different colors appeared upon two computer screens. To better simulate real-world choices, in which multiple options are available, children could earn "points" toward either their preferred AVG by clicking a computer mouse at one computer or toward their preferred SVG or TAP by clicking a computer mouse at the other computer. The child clicked the shapes until the shapes/colors matched, moving freely between the two screens as they chose. Matches earned them points toward their preferred activity. Initially, points were delivered after every four clicks, but then the schedule of reinforcement doubled $(4,8,16,32,[\ldots]$ 1024) each time five points were earned, so that ever-increasing amounts of work were required to earn more time in the desired activity. Each match earned one point, with points translating to minutes in each activity (in 5-minute increments). Children were permitted to play the game as long as they wished, earning as much time they were willing to play the game (either SVG, or AVG), up to a maximum of 45 minutes per game type. After completing the task, participants played their earned time of AVG and SVG, or earned time of AVG and most-liked TAP. AVG or SVG play took place in the laboratory, in single-player game mode.

Outcome measures included the breakpoint, or Pmax, ${ }^{31}$ which was the last schedule of reinforcement (i.e., 4, 8, $16, \ldots)$ completed for the behavior (AVG or SVG) and $\mathrm{RRV}_{\mathrm{AVG}}$ vs. SVG, which was calculated as $\left[\mathrm{Pmax}_{\mathrm{AVG}} /\right.$ $\left.\left(\operatorname{Pmax}_{\mathrm{AVG}}+\mathrm{Pmax}_{\mathrm{SVG}}\right)\right]$ and represents the RRV (amount of responding) of one behavior relative to the other. This final RRV score was considered an operationalization of AVG reinforcement. Similar methods of RRV of physical activity have been found to correlate with habitual physical exercise in both adults ${ }^{8,9}$ and children. ${ }^{7}$

Pmax and RRV $\mathrm{AVG}_{\text {vs. TAP. The }} \mathrm{RRV}_{\mathrm{AVG}}$ vs. TAP task followed a procedure identical to the $R R V_{A V G}$ vs. SVG reinforcement task, except that participants could earn time toward either AVG or TAP. TAP was completed indoors at a local fitness center and, to provide some context of the social aspect of the sport, the participant played against a member of the research team (K.U. or K.D.F.). Order of $\mathrm{RRV}_{\mathrm{AVG} \text { vs. SVG }}$ and $\mathrm{RRV}_{\mathrm{AVG}}$ vs. TAP was counterbalanced among participants.

\section{Analytic plan}

Baseline group differences for demographics, including gender, BMI, BMI percentile, and weight were tested using chi-squares and independent sample $t$-tests. Participants were blocked randomized on gender (male vs female), BMI (above 85th percentile vs. below 85th percentile), and 
Table 1. Baseline Demographics by Group

\begin{tabular}{lcc}
\hline & $\begin{array}{c}\text { Low autonomy, } \\
\mathrm{N}=25\end{array}$ & $\begin{array}{c}\text { High autonomy, } \\
\mathrm{N}=26\end{array}$ \\
\hline Age, years & $9.64 \pm 1.15$ & $10.12 \pm 1.18$ \\
Weight, kg & $39.43 \pm 10.76$ & $41.58 \pm 15.35$ \\
BMI, kg/m & $18.96 \pm 3.22$ & $18.91 \pm 4.08$ \\
BMI percentile & $65.66 \pm 26.28$ & $58.29 \pm 30.59$ \\
Gender, \% & & \\
$\quad$ Male & 21.57 & 27.45 \\
Female & 25.49 & 25.49 \\
\hline
\end{tabular}

Data are mean \pm SD.

No significant differences at baseline.

$\mathrm{BMI}$, body mass index; SD, standard deviation.

baseline liking of AVG ( 7 and lower vs. 8-10 on a 10-point scale) using Taves minimization.

The primary outcomes were conducted through a series of generalized linear mixed models because RRV data does not follow a normal distribution. $\operatorname{Pmax}_{\mathrm{AVG}}$, Pmax $\mathrm{SVG}_{\mathrm{SV}}$, Pmax $\mathrm{TAP}$, $\mathrm{RRV}_{\mathrm{AVG}}$ vs. SVG , and $\mathrm{RRV}_{\mathrm{AVG}}$ vs. TAP were each analyzed as the dependent variable in separate equations. For each equation, the between-subject variable was autonomy group (high vs. low autonomy), the within-subject variable was time point (baseline, 6 weeks, 10 weeks), and baseline RRV or Pmax scores were used as a covariate.

Based upon prior research and conservative estimates for multivariate effects, ${ }^{16,32}$ a prior power analysis suggested a sample size of $N=25$ per group to achieve power exceeding $1-\beta=0.90$. Because our final sample met this amount, it was assumed to have adequate power. All statistics were performed with SAS $9.4^{33}$ using Proc Glimmix, which allows for an intent-to-treat estimate of means. Major analyses were performed with an intent-to-treat analysis. However, because only two participants withdrew from the study following randomization, one from each autonomy condition, selective attrition is not believed to be of concern.

\section{Results}

Chi square and $t$-tests confirmed that autonomy groups did not differ at baseline in gender $(P=0.668)$, BMI percentile for age and gender $(P=0.362)$, BMI $(P=0.960)$, age $(P=$ $0.151)$, weight $(P=0.567)$ (Table 1$)$, as well as likings for AVG, SVG, and TAP at baseline, 6 weeks, and 10 weeks (Table 2). Participants' time per day spent in AVG, SVG, and TAP at baseline, 6 weeks, 10 weeks, as well as during the intervention itself are detailed in Table 3. Because participants did not play AVG every day, length of each session was also calculated, with the high autonomy group playing for an average of 64 minutes per session in the second week and 32 minutes per session in the fourth week. The low autonomy group played AVGs on an average of 32 minutes per session in the second week and 40 minutes per session in the fourth week.

\section{AVGs versus SVGs}

$\operatorname{Pmax}_{\mathrm{AVG}}$ showed an autonomy $\times$ time interaction $(P=$ $0.041)$. Specifically, for the high autonomy group, $P \max _{\mathrm{AVG}}$ decreased from baseline to 6 weeks and 6 to 10 weeks, whereas for the low autonomy group, Pmax ${ }_{\mathrm{AvG}}$ increased from baseline to 6 weeks and decreased from 6 to 10 weeks (Table 4). The effects of autonomy $(P=0.684)$ and time $(P=0.163)$ were not significant (Table 4$)$. There were no differences between groups at any time point by Tukey/Kramer contrasts $(P=0.118)$. Cross-group differences across time points also did not reach significance $(P=0.070)$.

Pmax $_{\text {SVG }}$ showed an effect of time $(P<0.001)$, but not autonomy $(P=0.836)$ or their interaction $(P=0.438)$. Pmax $_{\text {SVG }}$ decreased $57.8 \%(P<0.001)$ from baseline to 10 weeks. No changes were observed from baseline to 6 weeks $(-33.2 \%, P=0.139)$ or from 6 to 10 weeks $(-36.8 \%, P=$ 0.077). $\operatorname{RRV}_{\mathrm{AVG}}$ vs. SVG showed a borderline significant increase over time $(P=0.056)$, but there was no effect of autonomy $(P=0.578)$ or their interaction $(P=0.184)$. Across both autonomy groups, $\mathrm{RRV}_{\mathrm{AVG}}$ vs. SVG showed little change from baseline to 10 weeks $(P=0.072)$ (Table 5).

\section{AVGs versus TAP}

Pmax $_{\mathrm{AVG}}$ showed an effect of time $(P=0.001)$, but not autonomy $(P=0.590)$ or their interaction $(P=0.604)$. When tested against sport activities, Pmax $_{\mathrm{AVG}}$ decreased from baseline to 10 weeks $(P=0.007)$ and from 6 to 10 weeks $(P=0.002)$, but not baseline to 6 weeks $(P=0.926)$. Pmax $\operatorname{TaP}_{\mathrm{TAP}}$

Table 2. Liking Scores by Autonomy Group Across Time

\begin{tabular}{lcccc}
\hline Autonomy condition & Liking & Baseline & 6 Weeks & 10 Weeks \\
\hline AVG vs. SVG & & & & \\
$\quad$ High & AVG & $8.31(7.72-8.90)$ & $8.69(8.24-9.15)$ & $8.50(7.87-9.13)$ \\
$\quad$ Low & AVG & $8.21(7.50-8.91)$ & $8.36(7.70-9.03)$ & $8.13(7.41-8.85)$ \\
AVG vs. SVG & SVG & $8.54(7.76-9.32)$ & $8.03(7.07-9.00)$ & $7.96(7.03-8.89)$ \\
$\quad$ High & SVG & $8.50(7.72-9.28)$ & $8.50(7.69-9.31)$ & $7.91(6.98-8.84)$ \\
$\quad$ Low & AVG & $8.19(7.72-8.66)$ & $8.73(8.14-9.32)$ & $8.44(7.77-9.11)$ \\
AVG vs. TAP & AVG & $7.88(6.98-8.77)$ & $8.17(7.49-8.86)$ & $8.17(7.44-8.91)$ \\
$\quad$ High & TAP & $9.00(8.52-9.48)$ & $8.88(8.28-9.49)$ & $8.35(7.63-9.06)$ \\
$\quad$ Low & TAP & $7.96(6.97-8.95)$ & $7.91(7.02-8.81)$ & $7.74(6.79-8.69)$ \\
$\quad$ High & & & & \\
$\quad$ Low & &
\end{tabular}

Data are mean with $95 \%$ confidence interval.

No significant differences between autonomy groups at all time points.

AVG, active videogame; SVG, sedentary videogame; TAP, traditional active play. 
Table 3. Average Minutes Spent Per Day in Active Videogames, Sedentary Videogames, and Traditional Active Play by Time Points

\begin{tabular}{lcccccr}
\hline $\begin{array}{l}\text { Autonomy } \\
\text { group }\end{array}$ & Activity & $\begin{array}{c}\text { Baseline } \\
\text { (preintervention) }\end{array}$ & $\begin{array}{c}\text { Week 2 (during } \\
\text { intervention) }\end{array}$ & $\begin{array}{c}\text { Week 4 (during } \\
\text { intervention) }\end{array}$ & $\begin{array}{c}\text { Week 6 } \\
\text { (postintervention) }\end{array}$ & $\begin{array}{c}\text { Week 10 } \\
\text { (postwashout) }\end{array}$ \\
\hline High & AVG & $3 \pm 14$ & $32 \pm 74$ & $19 \pm 18$ & $19 \pm 27$ & $6 \pm 15$ \\
& SVG & $37 \pm 60$ & $17 \pm 32$ & $20 \pm 35$ & $21 \pm 35$ & $22 \pm 44$ \\
Low & TAP & $42 \pm 122$ & $35 \pm 50$ & $33 \pm 51$ & $54 \pm 192$ & $45 \pm 63$ \\
& AVG & $3 \pm 10$ & $17 \pm 19$ & $17 \pm 26$ & $35 \pm 25$ & $8 \pm 19$ \\
& SVG & $71 \pm 90$ & $25 \pm 50$ & $32 \pm 62$ & $29 \pm 60$ & $21 \pm 37$ \\
\hline
\end{tabular}

Data are mean \pm SD.

Data are from four randomly selected days (two weekend days and two weekdays) at each time point.

showed no effect of autonomy $(P=0.636)$, time $(P=0.593)$, or their interaction $(P=0.985)$ (Table 4$)$. $\mathrm{RRV}_{\mathrm{AVG}}$ vs. TAP showed a significant effect of time $(P=0.033)$, but not autonomy $(P=0.947)$ or their interaction $(P=0.727)$. Across both groups, $\mathrm{RRV}_{\mathrm{AVG}}$ vs. TAP showed a borderline significant decrease from baseline to 10 weeks $(P=0.058)$ and from 6 to 10 weeks $(P=0.059)$ (Table 5).

\section{Discussion}

Contrary to our predictions, exposure to AVGs did not increase SVG reinforcement in children. When completing the RRV $\mathrm{AVG}_{\text {vs. SVG }}$ task, reinforcement for both AVG and SVG declined at 6 and 10 weeks. Because SVG reinforcement decreased more, AVG reinforcement was greater relative to SVG postintervention. Declines in AVG reinforcement indicate that children were losing interest in the AVG games. This is not unexpected as others have demonstrated similar decline in AVG play once the novelty wears off, ${ }^{18}$ similar to the longitudinal decline in motivation common to other physical activity studies. ${ }^{19}$ However, these results indicate that AVG play decreases the RRV of SVG, a most encouraging result as sedentary behavior is implicated in increased BMI and poor health in both children ${ }^{34}$ and adults. ${ }^{35}$ Overall, these results indicate that parents, caretakers, and educators who wish to use AVGs to encourage children's active play need not worry about inadvertently increasing motivation to play SVG. Future research should investigate ways to slow declines in AVG interest, including a greater variety of games, encouraging group AVG play, and mixing AVG with TAP.

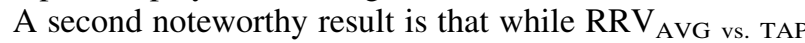
and $\mathrm{Pmax}_{\mathrm{AVG}}$ decreased, Pmax $\operatorname{maP}_{\mathrm{TAP}}$ showed no time effect.
While a null effect of Pmax $\operatorname{mAP}_{\text {TA }}$ may seem unnoteworthy, it stands in contrast to Pmax ${ }_{S V G}$ and Pmax $_{\mathrm{AVG}}$, which decreased. Therefore, $\mathrm{RRV}_{\mathrm{AVG}}$ vs. TAP decreased over time. This result could also be interpreted as the RRV of TAP $\left[\operatorname{Pmax}_{\mathrm{TAP}} /\left(\operatorname{Pmax}_{\mathrm{TAP}}+\mathrm{Pmax}_{\mathrm{AVG}}\right)\right]$ increasing relative to AVG, which argues that postintervention motivation to play TAP relative to AVGs was greater than at baseline. Because children often play active sports more intensely and expend comparatively more energy, ${ }^{16}$ increasing $\mathrm{RRV}_{\mathrm{TAP}}$ would promote this choice and the health of children. Our results suggest that AVGs may act as a gateway to TAP, an open question suggested by other studies. ${ }^{17}$ Studies with adult populations have shown similar results, in which AVG play leads to greater postintervention exercise behavior. ${ }^{14}$ Possibly, this occurs because playing AVG, rather increasing the RRV of AVG, decreases the RRV of SVG, which then makes alternatives, such as TAP, more appealing. Alternately, this result may be largely due to postintervention decreases in $\operatorname{Pmax}_{\mathrm{AVG}}$. Future research may wish to examine whether there is a tipping point that RRV of sedentary behavior must reach before RRV of physical activity, either AVG or TAP, increases, and if so, what factors may help sedentary individuals reach this point.

AVG play did not differentially change across autonomy groups. This stands in contrast to past findings, where greater autonomy, operationalized by choice of games, leads to longer play time. ${ }^{16}$ There are several potential explanations. Some of these discrepancies may be due to differences in laboratory ${ }^{16}$ versus home-based play, as other studies have noted that AVG play motivation depends on factors in the immediate enviroment. ${ }^{36}$ It is also possible that the construct of autonomy was not strongly varied to produce an autonomy

Table 4. Pmax Results by Autonomy Group and Time

\begin{tabular}{lccccc}
\hline Games compared & Pmax & Autonomy condition & Baseline & 6 Weeks & 10 Weeks \\
\hline AVG vs. SVG & AVG & High & $23.5(15.4-35.8)$ & $16.7(11.0-25.5)$ & $12.8(8.4-19.5)$ \\
& & Low & $14.4(9.4-22.2)$ & $27.1(17.6-41.7)$ & $16.6(10.7-25.7)$ \\
& SVG & High & $32.5(19.9-53.1)$ & $28.4(17.4-46.5)$ & $15.2(9.3-24.8)$ \\
& & Low & $39.4(23.8-65.1)$ & $20.2(12.2-33.3)$ & $15.0(9.0-24.9)$ \\
AVG vs. TAP & AVG & High & $19.0(12.4-29.1)$ & $22.9(15.0-35.1)$ & $12.2(7.9-18.6)$ \\
& & Low & $21.3(13.8-32.9)$ & $20.7(13.4-32.0)$ & $8.9(5.7-13.7)$ \\
& TAP & High & $28.1(17.1-46.3)$ & $25.0(15.2-41.1)$ & $23.3(14.2-38.3)$ \\
& & Low & $26.1(15.7-43.4)$ & $21.8(13.1-36.1)$ & $20.2(12.2-33.5)$ \\
\hline
\end{tabular}

Data are mean with $95 \%$ confidence interval.

Pmax scores are $\log _{2}$ transformed and covaried for baseline scores.

Pmax, last schedule of reinforcement completed. 
Table 5. Relative Reinforcing Value Results Across Both Groups AND TIME

\begin{tabular}{lcccc}
\hline $\begin{array}{l}\text { Games } \\
\text { compared }\end{array}$ & $\begin{array}{c}\text { Autonomy } \\
\text { condition }\end{array}$ & Baseline & 6 Weeks & 10 Weeks \\
\hline AVG vs. & High & $0.46 \pm 0.05$ & $0.46 \pm 0.05$ & $0.52 \pm 0.05$ \\
SVG & Low & $0.34 \pm 0.05$ & $0.52 \pm 0.05$ & $0.49 \pm 0.06$ \\
AVG vs. & High & $0.46 \pm 0.06$ & $0.50 \pm 0.06$ & $0.36 \pm 0.05$ \\
TAP & Low & $0.49 \pm 0.06$ & $0.45 \pm 0.06$ & $0.36 \pm 0.05$ \\
\hline
\end{tabular}

Data are mean \pm standard error.

effect or that any increases in AVG reinforcement engendered by the two games versus one diminished in less than 6 weeks. Some high autonomy participants may have preferentially played games from one disc only, thereby making their treatment similar with low autonomy participants. Even greater variety may have been needed for the effects of autonomy to last long term. Furthermore, although the high autonomy group chose longer AVG sessions early in the intervention, by the intervention midpoint, the average session time had decreased until sessions were no longer than the time allotted to the low autonomy group (Table 3).

Another possibility is that other factors were needed to increase AVG reinforcement. During the RRV task, children played AVGs against the computer rather than an opponent. Many videogame systems allow the option of a single-player game against the computer or multiplayer games against siblings, friends, or even unknown individuals from online. Motivation toward AVGs is sustained more in multiplayer compared with individual player games, ${ }^{37,38}$ suggesting a social aspect to AVG play consistent with the social aspects of other forms of exercise. This social aspect may also extend to TAP. During the current study, children played TAP with a member of the research team, which may have served to make the TAP option more appealing compared with AVG. Furthermore, this laboratory-based testing situation was an artificial social situation, so suggesting that the social appeal of TAP may have been even greater in TAP play with siblings, friends, or parents. Motivation to be physically active is greater with a friend even within an artificial laboratory setting $^{39}$ and physical activity intensity tends to be greater in the presence of peers, ${ }^{40}$ suggesting that the social context may be a critical factor in encouraging AVG and TAP.

This study has additional limitations. Our study was of limited duration; long-term exercise reinforcement over the course of months is necessary for establishing life-long healthy habits. Furthermore, these results may not generalize to other age groups, including younger children and adults. Although traditional exercise results in greater energy expenditure, adults rate AVGs as more enjoyable. ${ }^{41}$ Given the well-known decrease in physical activity during the adolescent years, future studies may wish to investigate the ability of AVGs to increase exercise reinforcement during this developmental phase. Our results also may vary based upon the types of games offered, including both videogame types and TAPs. It is possible that children would have responded differently had their preferred game/sport or a team-based context been offered. Furthermore, a wide variety of AVG systems and games are currently on the market. Energy expenditure, enjoyment, and other characteristics relevant for continued long-term usage vary between games and systems,${ }^{42-44}$ which suggests that results from a study utilizing Xbox ${ }^{\circledR}$ Kinects ${ }^{\mathrm{TM}}$ may not generalize to other systems or games. Although low and high autonomy groups showed different patterns of $\mathrm{Pmax}_{\mathrm{AVG}}$ when playing for AVG versus SVG, these discrepancies may have been largely driven by baseline differences across groups (Table 3). Future studies may wish to block based upon baseline Pmax scores, rather than baseline liking, which showed little variation.

In summary, providing inactive children with AVGs may be used as a method to increase their active play reinforcement. Despite concerns, increased AVG reinforcement does not increase but rather decreases relative reinforcement of SVG. Furthermore, AVG reinforcement does not displace TAP reinforcement, which remains robust even when paired with AVG play. Increased autonomy through greater game choice does not increase AVG play reinforcement and reinforcement of AVGs tends to decline over time; future research must examine other factors, which might help maintain AVG play long term.

\section{Acknowledgments}

The authors would like to thank Bill Siders, Doreen Rolshoven, Colton Peltier, Ethan Brown, as well as our research volunteers and their families for their time and assistance.

\section{Author Disclosure Statement}

No competing financial interests exist.

\section{Funding Information}

This work was funded by the United States Department of Agriculture, Agricultural Research Service, 3062-51000-5100D. The mention of trade names, commercial products, or organizations does not imply endorsement from the U.S. government. USDA is an equal opportunity provider and employer.

\section{References}

1. U.S. Department of Health and Human Services. Physical Activity Guidelines for Americans. Washington, DC: U.S. Department of Health and Human Services; 2008:15-34.

2. Kann L, McManus T, Harris WA, et al. Youth risk behavior surveillance-United States, 2017. MMWR Surveill Summ 2018; 67:1-114.

3. Katzmarzyk PT, Lee I-M, Martin CK, Blair SN. Epidemiology of physical activity and exercise training in the United States. Prog Cardiovasc Dis 2017; 60:3-10.

4. Kokkinos P, Sheriff H, Kheirbek R. Physical inactivity and mortality risk. Cardiol Res Pract 2011; 2011:924945.

5. Epstein LH, Roemmich JN. Reducing sedentary behavior: Role in modifying physical activity. Exerc Sport Sci Rev 2001; 29:103-108.

6. Epstein LH, Kilanowski CK, Consalvi AR, Paluch RA. Reinforcing value of physical activity as a determinant of child activity level. Health Psychol 1999; 18:599.

7. Barkley JE, Epstein LH, Roemmich JN. Reinforcing value of interval and continuous physical activity in children. Physiol Behav 2009; 98:31-36.

8. Flack KD, Johnson L, Roemmich JN. Aerobic and resistance exercise reinforcement and discomfort tolerance predict meeting activity guidelines. Physiol Behav 2017; 170:32-36.

9. Flack KD, Johnson L, Roemmich JN. The reinforcing value and liking of resistance training and aerobic exercise as 
predictors of adult's physical activity. Physiol Behav 2017; 179:284-289.

10. Smits-Engelsman BC, Jelsma LD, Ferguson GD. The effect of exergames on functional strength, anaerobic fitness, balance and agility in children with and without motor coordination difficulties living in low-income communities. Hum Mov Sci 2017; 55:327-337.

11. Wagener T, Fedele D, Mignogna M, et al. Psychological effects of dance-based group exergaming in obese adolescents. Pediatr Obes 2012; 7:e68-e74.

12. Graf DL, Pratt LV, Hester CN, Short KR. Playing active video games increases energy expenditure in children. Pediatrics 2009; 124:534-540.

13. Baranowski T, Abdelsamad D, Baranowski J, et al. Impact of an active video game on healthy children's physical activity. Pediatrics 2012; 129:e636.

14. Bock BC, Dunsiger SI, Ciccolo JT, et al. Exercise videogames, physical activity, and health: Wii heart fitness: A randomized clinical trial. Am J Prev Med 2019; 56: 501-511.

15. Biddiss E, Irwin J. Active video games to promote physical activity in children and youth: A systematic review. Arch Pediatr Adolesc Med 2010; 164:664-672.

16. Roemmich JN, Lambiase MJ, McCarthy TF, et al. Autonomy supportive environments and mastery as basic factors to motivate physical activity in children: A controlled laboratory study. Int J Behav Nutr Phys Act 2012; 9:16.

17. Baranowski T. Exergaming: Hope for future physical activity? or blight on mankind? J Sport Health Sci 2017; 6:44.

18. Barnett A, Cerin E, Baranowski T. Active video games for youth: A systematic review. J Phys Act Health 2011; 8:724-737.

19. Sallis JF, Haskell WL, Fortmann SP, et al. Predictors of adoption and maintenance of physical activity in a community sample. Prev Med 1986; 15:331-341.

20. Chatzisarantis NL, Hagger MS, Smith B. Influences of perceived autonomy support on physical activity within the theory of planned behavior. Eur J Soc Psychol 2007; 37:934-954.

21. González-Cutre D, Ferriz R, Beltrán-Carrillo VJ, et al. Promotion of autonomy for participation in physical activity: A study based on the trans-contextual model of motivation. Educ Psychol 2014; 34:367-384.

22. Sanders GJ, Juvancic-Heltzel J, Williamson ML, et al. The effect of increasing autonomy through choice on young children's physical activity behavior. J Phys Act Health 2016; 13:428-432.

23. Feda DM, Lambiase MJ, McCarthy TF, et al. Effect of increasing the choice of active options on children's physically active play. J Sci Med Sport 2012; 15:334-340.

24. Gunnell KE, Bélanger M, Brunet J. A tale of two models: Changes in psychological need satisfaction and physical activity over 3 years. Health Psychol 2016; 35:167.

25. Fenton SA, Duda JL, Quested E, Barrett T. Coach autonomy support predicts autonomous motivation and daily moderate-to-vigorous physical activity and sedentary time in youth sport participants. Psychol Sport Exerc 2014; 15: 453-463.

26. Barkley JE, Ryan EJ, Bellar D, et al. The variety of exercise equipment and physical activity participation in children. J Sport Behav 2011; 34:137.

27. Anderson SE, Economos CD, Must A. Active play and screen time in US children aged 4 to 11 years in relation to sociodemographic and weight status characteristics: A nationally representative cross-sectional analysis. BMC Public Health 2008; 8:366.
28. Simons M, de Vet E, Hoornstra S, et al. Adolescents' views on active and non-active videogames: A focus group study. Games Health J 2012; 1:211-218.

29. Kuczmarski, RJ, Ogden CL, Guo SS et al., 2000 CDC Growth Charts for the United States: Methods and development. Vital Natl Health Stat 2002;11:246.

30. Simons M, Chinapaw MJ, Brug J, et al. Associations between active video gaming and other energy-balance related behaviours in adolescents: A 24-hour recall diary study. Int J Behav Nutr Phys Act 2015; 12:32.

31. Bickel WK, Marsch LA, Carroll ME. Deconstructing relative reinforcing efficacy and situating the measures of pharmacological reinforcement with behavioral economics: A theoretical proposal. Psychopharmacology 2000; 153:44-56.

32. Cohen J. A power primer. Psychol Bull 1992; 112:155.

33. SAS Institute. Base SAS 9.4 Procedures Guide. Cary, NC: SAS Institute; 2015.

34. Must A, Tybor D. Physical activity and sedentary behavior: A review of longitudinal studies of weight and adiposity in youth. Int J Obes 2005; 29:S84.

35. Ford ES, Kohl HW, Mokdad AH, Ajani UA. Sedentary behavior, physical activity, and the metabolic syndrome among US adults. Obesity 2005; 13:608-614.

36. Robertson J, Jepson R, MacVean A, Gray S. Understanding the importance of context: A qualitative study of a locationbased exergame to enhance school childrens physical activity. PLoS One 2016; 11:e0160927.

37. Peng W, Crouse J. Playing in parallel: The effects of multiplayer modes in active video game on motivation and physical exertion. Cyberpsychol Behav Soc Netw 2013; 16:423-427.

38. Paw MJCA, Jacobs WM, Vaessen EP, et al. The motivation of children to play an active video game. J Sci Med Sport 2008; 11:163-166.

39. Salvy S-J, Roemmich JN, Bowker JC, et al. Effect of peers and friends on youth physical activity and motivation to be physically active. J Pediatr Psychol 2008; 34:217-225.

40. Salvy S-J, Bowker JW, Roemmich JN, et al. Peer influence on children's physical activity: An experience sampling study. J Pediatr Psychol 2007; 33:39-49.

41. McDonough D, Pope Z, Zeng N, et al. Comparison of college students' energy expenditure, physical activity, and enjoyment during exergaming and traditional exercise. J Clin Med 2018; 7:433.

42. Sanders GJ, Peacock CA, Barkley JE, et al. Heart rate and liking during "Kinect Boxing" versus "Wii Boxing": The potential for enjoyable vigorous physical activity videogames. Games Health J 2015; 4:265-270.

43. Parent A-A, Comtois A-S. Comparison of various active video gameplays and consoles on the energy expenditure and cardiorespiratory responses in young male adult gamers. Entertain Comput 2019; 30:100293.

44. Peng W, Lin J-H, Crouse J. Is playing exergames really exercising? A meta-analysis of energy expenditure in active video games. Cyberpsychol Behav Soc Netw 2011; 14: 681-688.

Address correspondence to: Kelsey Ufholz, PhD

United States Department of Agriculture Agricultural Research Service 2420 2nd Avenue North Grand Forks, ND 58203

E-mail: kelsey.ufholz@ars.usda.gov 